\title{
Proximity-Induced Odd-Frequency Superconductivity in a Topological Insulator
}

\author{
Jonas A. Krieger $\odot,{ }^{1,2,3}$ Anna Pertsova $\odot,{ }^{4}$ Sean R. Giblin, ${ }^{5}$ Max Döbeli, ${ }^{6}$ Thomas Prokscha $\odot,{ }^{1}$ Christof W. Schneider $\odot,{ }^{7}$ \\ Andreas Suter@, ${ }^{1}$ Thorsten Hesjedal@ ${ }^{8}$ Alexander V. Balatsky, ${ }^{4,9,}$ and Zaher Salman ${ }^{1, \dagger}$ \\ ${ }^{1}$ Laboratory for Muon Spin Spectroscopy, Paul Scherrer Institute, CH-5232 Villigen PSI, Switzerland \\ ${ }^{2}$ Laboratorium für Festkörperphysik, ETH Zürich, CH-8093 Zürich, Switzerland \\ ${ }^{3}$ Swiss Light Source, Paul Scherrer Institute, CH-5232 Villigen PSI, Switzerland \\ ${ }^{4}$ Nordita, Roslagstullsbacken 23, SE-106 91 Stockholm, Sweden \\ ${ }^{5}$ School of Physics and Astronomy, Cardiff University, Cardiff CF24 3AA, United Kingdom \\ ${ }^{6}$ Ion Beam Physics, ETH Zürich, Otto-Stern-Weg 5, CH-8093 Zürich, Switzerland \\ ${ }^{7}$ Laboratory for Multiscale Materials Experiments, Paul Scherrer Institute, CH-5232 Villigen PSI, Switzerland \\ ${ }^{8}$ Department of Physics, Clarendon Laboratory, University of Oxford, Oxford OX1 3PU, United Kingdom \\ ${ }^{9}$ Department of Physics, University of Connecticut, Storrs, Connecticut 06268, USA
}

(Received 16 March 2020; accepted 16 June 2020; published 10 July 2020)

\begin{abstract}
At an interface between a topological insulator (TI) and a conventional superconductor (SC), superconductivity has been predicted to change dramatically and exhibit novel correlations. In particular, the induced superconductivity by an $s$-wave SC in a TI can develop an order parameter with a $p$-wave component. Here we present experimental evidence for an unexpected proximity-induced novel superconducting state in a thin layer of the prototypical $\mathrm{TI}, \mathrm{Bi}_{2} \mathrm{Se}_{3}$ proximity coupled to $\mathrm{Nb}$. From depthresolved magnetic field measurements below the superconducting transition temperature of $\mathrm{Nb}$, we observe a local enhancement of the magnetic field in $\mathrm{Bi}_{2} \mathrm{Se}_{3}$ that exceeds the externally applied field, thus supporting the existence of an intrinsic paramagnetic Meissner effect arising from an odd-frequency superconducting state. Our experimental results are complemented by theoretical calculations supporting the appearance of such a component at the interface which extends into the TI. This state is topologically distinct from the conventional Bardeen-Cooper-Schrieffer state it originates from. To the best of our knowledge, these findings present a first observation of bulk odd-frequency superconductivity in a TI. We thus reaffirm the potential of the TI-SC interface as a versatile platform to produce novel superconducting states.
\end{abstract}

DOI: 10.1103/PhysRevLett.125.026802

In a conventional superconductor (SC), the electronic excitations are usually described as condensation of Cooper pairs [1]. Fermi statistics imply symmetry constraints on permutation properties of the pair wave function, thus limiting the possible SC classes [2]. According to conventional classification, states with even parity $(s, d$ wave) must be in a spin-singlet configuration while states with odd parity ( $p, f$ wave) must be in a spin-triplet configuration. However, additional classes are possible when permutation with respect to time and, if present, orbital degrees of freedom are included. This general classification allows for the odd-frequency, or Berezinskii, state characterized by superconducting pairing which is nonlocal and odd in time [2-6]. Odd-frequency pairing gives rise to SC

Published by the American Physical Society under the terms of the Creative Commons Attribution 4.0 International license. Further distribution of this work must maintain attribution to the author(s) and the published article's title, journal citation, and DOI. states with symmetries different from conventional states, for example triplet $s$-wave $[3,4]$ and singlet $p$-wave [5] states. The Berezinskii state is currently recognized as an inherently dynamical order that can be realized in a variety of systems, including bulk SCs, heterostructures, and dynamically driven systems. It is especially relevant at interfaces, where locally broken symmetries can influence the type of pairing $[6,7]$.

One of the peculiarities of such a state is that the sign of the Meissner screening can be reversed in some cases, causing an attraction instead of a repulsion of external magnetic fields. Paramagnetic Meissner response has been predicted in proximity structures $[6,8-12]$ and in multiband SCs [13]. While such a paramagnetic Meissner screening cannot be stable in the bulk, it has been observed at interfaces, where the superconductivity is induced in a nonsuperconducting layer by the proximity effect [14]. One particular superconducting interface that has attracted significant attention in recent years is between a conventional $s$-wave SC and a 3D topological insulator (TI). Fu and Kane have predicted that induced superconductivity in 

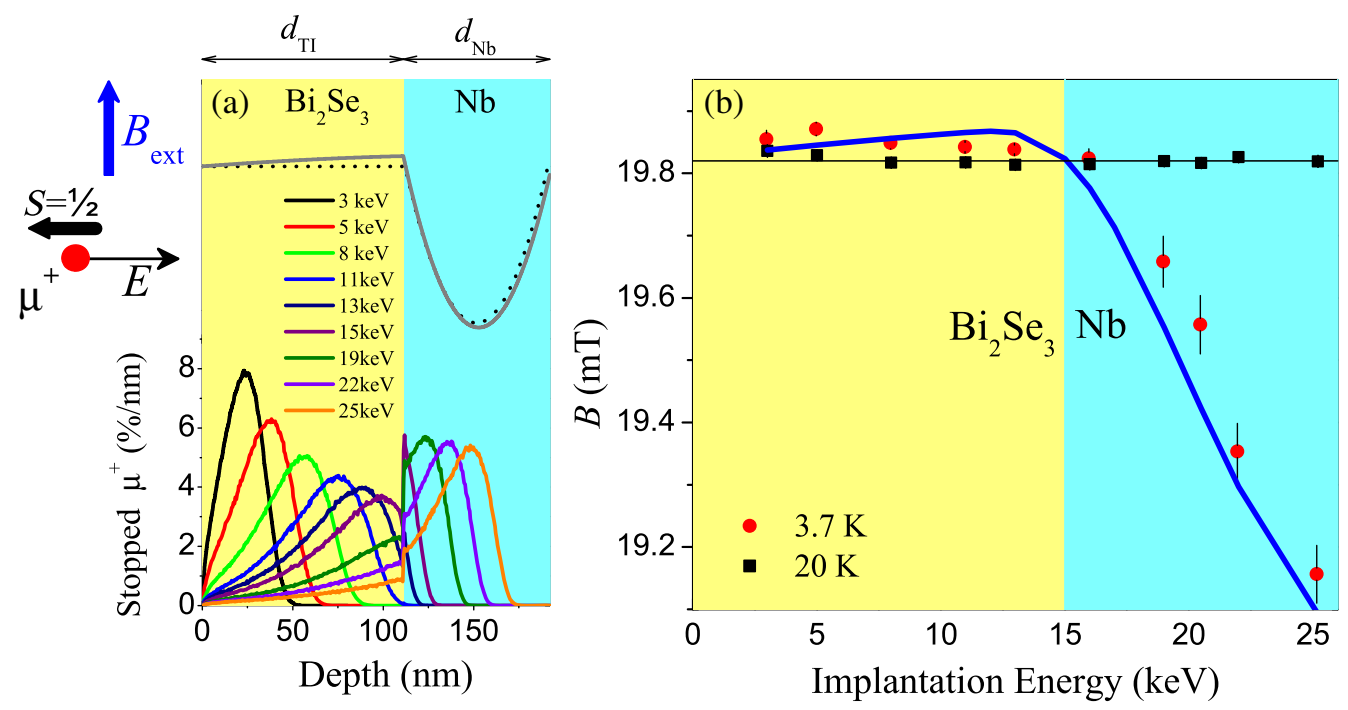

FIG. 1. (a) (top) Schematic showing the $\mu$ SR geometry where the applied field is parallel to the surface and perpendicular to the muon spin and momentum directions. The lines depict the expected magnetic field depth profile due to screening of the applied field inside the heterostructure with (solid gray) and without (dotted black) proximity induced superconductivity in the $\mathrm{Bi}_{2} \mathrm{Se}_{3}$ layer. (bottom) Calculated muon implantation profiles at different implantation energies using Trim.SP [25]. (b) Measured local mean field as a function of implantation energy above (black squares) and below (red circles) the superconducting transition temperature. The black horizontal line represents the applied field value, $B_{\text {ext }}$, and the blue line is a fit to the theoretical model (see text).

the topological surface state (TSS) may have a $p_{x}+i p_{y}$ even-frequency order parameter, that might allow for the stabilization of Majorana bound states in vortex cores [15]. The latter are the key ingredient in a proposal for faulttolerant quantum computation. Furthermore, the occurrence of Majorana zero modes can be related to the presence of odd-frequency superconducting components [16-19]. Aside from the conventional even-frequency superconductivity, one should be on a lookout for oddfrequency correlations induced at such interfaces.

In this Letter, we provide experimental evidence of proximity induced odd-frequency superconductivity in a heterostructure of $\mathrm{Bi}_{2} \mathrm{Se}_{3}(\sim 110 \mathrm{~nm})$ on $\mathrm{Nb}(\sim 80 \mathrm{~nm})$. In particular, we measure the depth dependence of the magnetic field parallel to the interface in the Meissner state using low energy muon spin rotation [20-23] (LE- $\mu \mathrm{SR})$ at the $\mu \mathrm{E} 4$ beam line [24] of the Swiss Muon Source at PSI, Switzerland. This technique allows for a high precision characterization of the magnetic field profile by measuring the average Larmor precession frequency of the muons' spins as a function of their implantation energy (and corresponding implantation depth), as illustrated in Fig. 1(a). Below the superconducting transition temperature of $\mathrm{Nb}$ we observe conventional diamagnetic Meissner screening in the $\mathrm{Nb}$ layer. In contrast, a paramagnetic Meissner screening is observed in the $\mathrm{Bi}_{2} \mathrm{Se}_{3}$ layer, indicating a proximity-induced odd-frequency superconducting component in the TI layer. Our experimental results are complemented by theoretical calculations supporting the appearance of such an odd-frequency component at the interface, extending deep into the TI.
The local magnetic field as a function of implantation energy, $E$, is shown in Fig. 1(b). At a temperature of $20 \mathrm{~K}$, i.e., in the normal state, the muons probe a depthindependent magnetic field (black squares). At temperatures below the superconducting transition of $\mathrm{Nb}$, we observe a strong variation in the measured field as a function of depth, featuring a typical Meissner screening inside the $\mathrm{Nb}$ layer (red circles). In a conventional metal-SC proximity structure, the induced superfluid density in the metal results in a decreased mean field which increases monotonically as a function of distance from the metal-SC interface, reaching the applied field value far inside the metal [26-28]. In contrast, the behavior in $\mathrm{Bi}_{2} \mathrm{Se}_{3} / \mathrm{Nb}$ is reversed; the field in the TI is enhanced compared to the applied field value, $B_{\text {ext }}$ (i.e., its value at $20 \mathrm{~K}$ ). This can be clearly seen in the fast Fourier transform of the muon spin polarization spectra which represent the local field distribution sensed by the muons [Fig. 2(a)]. A careful inspection of the temperature dependence of this effect shows that the paramagnetic field shift in $\mathrm{Bi}_{2} \mathrm{Se}_{3}$ occurs below the superconducting transition temperature, $T_{c} \approx 9 \mathrm{~K}$, of $\mathrm{Nb}$ [Fig. 2(b)]. This is in agreement with $T_{c}$ obtained from transport measurements which show a sharp superconducting transition below $9 \mathrm{~K}$ (see the Supplemental Material [29]). We have also measured the magnetic field as a function of depth for various $B_{\text {ext }}$ values in the range 7 to $20 \mathrm{mT}$. We find that the local field at low temperatures is proportional to the applied field, always exhibiting a paramagnetic shift inside the TI. This indicates that the induced superfluid density is almost field-independent within this field range (see the Supplemental Material [29]). 

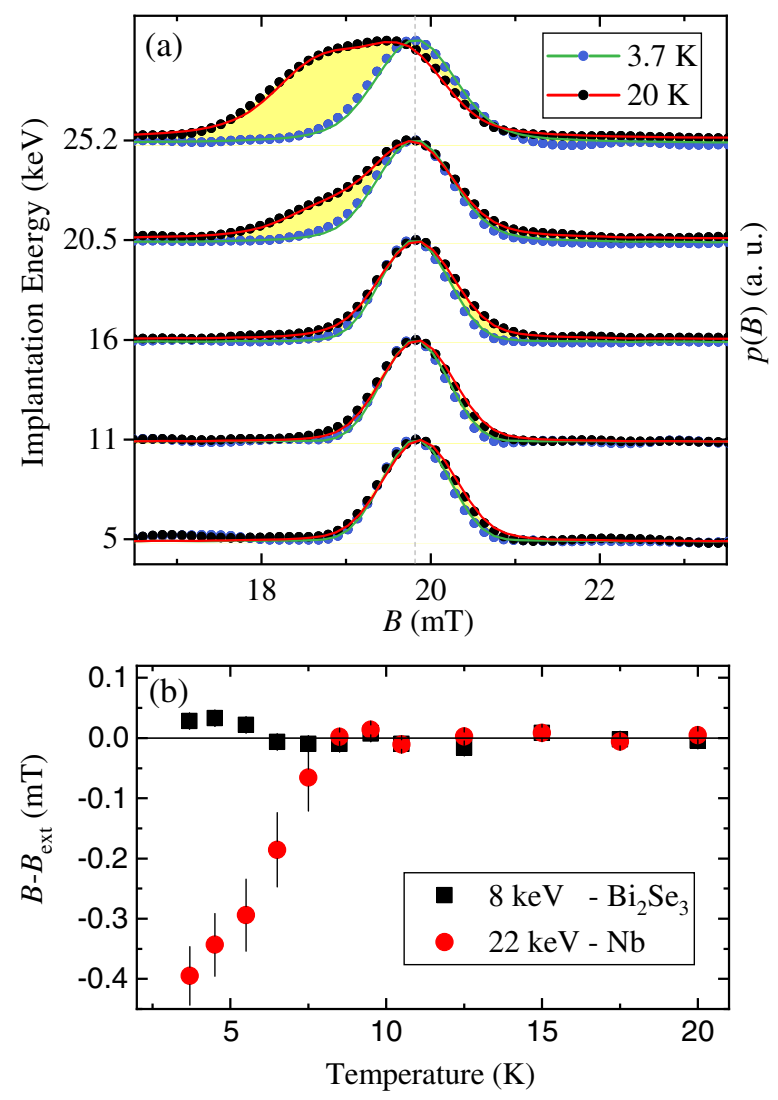

FIG. 2. (a) Local field distribution calculated from fast Fourier transform of the muon polarization measured in the heterostructure, above and below the superconducting transition temperature. Different energies are offset for clarity. The solid lines represent results from the fitting procedure (see the Supplemental Material [29]). (b) Temperature dependence of the field shift in the $\mathrm{Bi}_{2} \mathrm{Se}_{3}$ and $\mathrm{Nb}$ layers.

The observed paramagnetic shift of about $0.2 \%$ is too large to be attributed to the demagnetization fields of the $\mathrm{Nb}$ layer, which in a thin film are only relevant close to the edges [29]. Furthermore, a misalignment of the applied field with respect to the interface could only reduce the mean field measured in the sample. Another possible source for a positive shift are microscopic demagnetization fields caused by the roughness of the SC-TI interface. However, such stray fields should decrease exponentially with distance away from the interface [40,41], which does not agree with our observation. We can also exclude a temperature dependence of the field in bulk $\mathrm{Bi}_{2} \mathrm{Se}_{3}$ or any systematic deviations caused by the experimental setup [42].

Odd-frequency components in superconductors are expected at any ballistic interface [7] and in TIs, they may also occur in the presence of in-plane gap gradients [43], exchange fields [44] and multigap odd-orbital coupling [38]. However, they are usually a subdominant component compared to the even-frequency pairs. The total shielding current given by $\mathbf{j}=-e^{2}\left(n_{e}-n_{o}\right) \mathbf{A} / m c$, where $n_{e}$ and $n_{o}$ are the superfluid densities of the even and odd pairs, respectively, and A the vector-potential, should therefore still be diamagnetic [11]. The observed extension of the positive shift deep into the $\mathrm{Bi}_{2} \mathrm{Se}_{3}$ layer, shown in Fig. 1(b), is a clear indication that not only the TSS but also the bulk conduction band of $\mathrm{Bi}_{2} \mathrm{Se}_{3}$ is relevant for the proximity effect, as has also been pointed out by previous studies [45]. Therefore, we conclude that the observed paramagnetic Meissner screening is due to supercarriers induced into the bulk conduction band of $\mathrm{Bi}_{2} \mathrm{Se}_{3}$ by the proximity to superconducting $\mathrm{Nb}$. Furthermore, this observation suggests that the proximity-induced superconducting state is the unconventional odd-frequency (Berezinskii) state. Such a nontrivial pairing state in TIs has not been observed before.

To make a better comparison and illustrate the veracity of our conclusion we provide a summary of theoretical calculations that support our experimental results. We have developed a theoretical description of proximity-induced superconductivity in the TI-SC heterostructure based on a well-established two-orbital tight-binding model for $\mathrm{Bi}_{2} \mathrm{Se}_{3}$ $[38,46]$ (see the Supplemental Material [29]). We show that the induced odd-frequency pairing persists in the bulk of the TI, and that it dominates the Meissner effect near the TI-SC interface. Based on the insights from this microscopic model, we propose a theoretical depth profile of the magnetic field, derived from Maxwell's equations and linear response theory, that quantitatively fits our experimental data.

The band inversion in $\mathrm{Bi}_{2} \mathrm{Se}_{3}$ implies that the bulk conduction and valence bands are formed by two orbitals with different parity, originating from hybridized $\mathrm{Se}$ and $\mathrm{Bi}$ $p_{z}$ states. This orbital degree of freedom in the TI allows for the generation of odd-frequency pairing components, in addition to the even-frequency ones [47]. For a proximitycoupled TI to a singlet $s$-wave SC, the symmetry allowed odd- (even-) frequency components are odd (even) in the orbital index [38]. In addition to the dominant $s$-wave singlet pairing, even- and odd-frequency $p$-wave triplet components are present. Our calculations show strong oddfrequency SC correlations that propagate away from the interface due to the coupling between $\mathrm{Nb}$ and the bulk electronic states of $\mathrm{Bi}_{2} \mathrm{Se}_{3}$. The two orbitals in the tightbinding model correspond to top and bottom Se $p_{z}$ states in a quintuple layer, each hybridized with the neighboring $\mathrm{Bi}$ atoms [48]. We assume stronger tunneling from the SC into the orbital closest to the interface. This gives rise to oddfrequency components that can be comparable or larger in magnitude than the even-frequency ones over a wide range of frequencies. Details of this effect and its depth dependence are given in the Supplemental Material [29].

The penetration depth of the induced SC pairing in the TI layer depends on the position of the chemical potential. When the chemical potential is at the Dirac point of the TI, the induced SC pairing amplitudes decay within approximately 
two quintuple layers from the interface, i.e., a typical penetration depth of the TI surface states. However, when the chemical potential is in the bulk conduction band, as is the case for the (intrinsically) $n$-doped $\mathrm{Bi}_{2} \mathrm{Se}_{3}$ sample used here, we find finite SC pairing amplitudes far away from the interface. We attribute this to coupling between the SC and bulk TI states. This is consistent with the experimental observation of the Meissner screening inside the TI layer shown in Fig. 1(b). The role of bulk states in the TI-SC heterostructure has been previously pointed out for trivial even-frequency pairing [45]. We extend this approach to allow for odd-frequency pairing induced in the TI.

We will now focus on the interpretation of the positive field shift in the observed Meissner screening. In ordinary metals or semiconductors, odd-frequency pairing leads to the paramagnetic Meissner effect [13]. Recently, it has been pointed out that in materials with Dirac dispersion, interband contributions to the current-current correlation function can give rise to a diamagnetic Meissner response [49]. This has been extended to a low-energy model of a doped 3D TI, where it was found that odd-frequency pairing can give rise to a diamagnetic response due to interband effects [50]. More generally, the total magnetic response can be para- or diamagnetic depending on the balance between intra- and interband processes [50]. This complication, however, may be less relevant for the system considered here since the model of a bulk TI does not rely on the Dirac Hamiltonian. Therefore, we attribute the paramagnetic shift to the odd-frequency components in the bulk TI. Based on these considerations, we developed a phenomenological model of the Meissner effect.

We consider a TI-SC heterostructure with the interface at $z=0, \mathrm{SC}(\mathrm{Nb})$ extending from $-d_{\mathrm{SC}}$ to 0 and the $\mathrm{TI}$ $\left(\mathrm{Bi}_{2} \mathrm{Se}_{3}\right)$ from 0 to $d_{\mathrm{TI}}$. Similarly to Ref. [14], we solve a differential equation for the vector potential $A_{x}(z) \equiv A(z)$

$$
\frac{d^{2} A(z)}{d z^{2}}=K_{x x}(z) A(z)
$$

where $K_{x x}(z)$ is the current-current correlation function, or the Meissner kernel, which determines the magnetic response of the system. The local magnetic field is calculated as $B(z)=d A(z) / d z$. We use matching boundary conditions for $A(z)$ and $B(z)$ at the TI-SC interface and set $B(z)=B_{\text {ext }}$ outside the heterostructure. Furthermore, we propose the following form of the Meissner kernel in the TI and SC layers

$$
K_{x x}(z)= \begin{cases}\frac{1}{\lambda_{\mathrm{SC}}^{2}} & -d_{\mathrm{SC}}<z<0 \\ -\frac{1}{\lambda_{\mathrm{TI}}^{2}} e^{-2 z / z_{0}} & 0<z<d_{\mathrm{TI}},\end{cases}
$$

where $\lambda_{\mathrm{SC}}$ is the London penetration depth of the SC while $\lambda_{\mathrm{TI}}$ and $z_{0}$ are the characteristic length scales in the TI layer. Thus, we assume a conventional diamagnetic Meissner screening in the SC, which leads to exponential suppression of the local magnetic field extending into the SC layer. On the TI side, the kernel describes the proximity-induced odd-frequency screening which exponentially decays over a persistence length $z_{0}$ from the TI-SC interface.

We solve Eq. (1) with the kernel $K_{x x}$ given in Eq. (2) and with boundary conditions at all three interfaces to obtain the theoretical depth profile of the local magnetic field, $B_{\text {loc }}^{\text {theo }}(z)$. To fit the experimental data, we use $B_{\text {loc }}^{\text {theo }}(z)$ to calculate the muon precession signal averaged over its stopping distribution at a given $E$ (see details in the Supplemental Material [29]). The minimization of the fit was performed for all $E$ values (at $T=3.7 \mathrm{~K}$ ) simultaneously, using the same theoretical field profile. We also fixed the thickness of the layers obtained from RBS and XRR measurements, $d_{\mathrm{TI}}=$ $111.1 \mathrm{~nm}$ and $d_{\mathrm{SC}}=81 \mathrm{~nm}$, together with the applied field obtained from the measurements at $T=20 \mathrm{~K}$, $B_{\text {ext }}=19.818(2) \mathrm{mT}$. The parameters extracted from this global fit [gray line in Fig. 1(a)] give $\lambda_{\mathrm{TI}}=1.62(4) \mu \mathrm{m}$ and $\lambda_{\mathrm{SC}}=117.8(6) \mathrm{nm}$. In this fit $z_{0}=1 \mu \mathrm{m}$ is chosen to reflect the fact that superconductivity is induced in the $\mathrm{Bi}_{2} \mathrm{Se}_{3}$ bulk conductance states above the TI gap and, therefore, has a long decay range inside the TI. We checked that the specific choice of $z_{0}$ does not affect the quality of the fit. However, the exact value of $\lambda_{\text {TI }}$ systematically depends on the choice of $z_{0}$ and varies between $0.90(2)$ and $1.74(5) \mu \mathrm{m}$ for $z_{0}$ within $\left[d_{\mathrm{TI}}\right.$, $\infty)$. We assume that the the penetration depth in $\mathrm{Nb} \lambda_{\mathrm{SC}}$ is not modified significantly by the interface. However, note that the obtained value is much larger than that in the clean limit of $\mathrm{Nb}$ [27(3) $\mathrm{nm}[21]]$. Finally, we calculated the mean local field as a function of $E$ by averaging $B_{\mathrm{loc}}^{\text {theo }}(z)$ over the corresponding muons' stopping distribution. This is plotted in Fig. 1(b) (blue line) and exhibits a very good agreement with the mean field obtained independently, confirming again the validity of the obtained $B_{\mathrm{loc}}^{\text {theo }}(z)$.

Hence, the proposed theoretical field profile, based on the assumption of a large odd-frequency pairing amplitude in the bulk of the TI supported by the microscopic model, explains qualitatively and quantitatively the observed paramagnetic Meissner shift. The measured local field profile shows an almost constant paramagnetic shift in the TI extending at least $30 \mathrm{~nm}$ from the TI-SC interface and a conventional screening on the superconducting $\mathrm{Nb}$ side. The paramagnetic shift decreases gradually towards the opposite TI surface. Deviations of the experimental data from the predicted profile, for example a small positive shift of the mean magnetic field above $B_{\text {ext }}$ on the SC side just below the interface may be due to the limited accuracy in calculating the stopping profiles using Trim.SP [Fig. 1(a)]. Furthermore, some of the experimental details that may affect the local field profile, such as interface roughness and inhomogeneous thickness of the layers, are not included in the theoretical modeling. However, we except this to have a negligible effect, since the roughness is on an the length scale of one quintuple layer $(\sim 1.4 \mathrm{~nm})$ [29]. Therefore, the magnetic field response is set by the macroscopic length 
scales, such as the penetration depth. We also point out that both the induced dominant odd-frequency superconductivity and the underlying $\mathrm{Nb}$ superconductivity are of $s$-wave type. Therefore the effects of atomic disorder at the interface are not likely to degrade the induced odd-frequency state.

In conclusion, we observe an intrinsic paramagnetic Meissner shift in proximity-induced superconductivity in a $\mathrm{Bi}_{2} \mathrm{Se}_{3} / \mathrm{Nb}$ heterostructure. We attribute this effect to odd-frequency superconductivity which persists up to tens of nanometers away from the $\mathrm{Bi}_{2} \mathrm{Se}_{3} / \mathrm{Nb}$ interface. This finding, which is supported by our theoretical calculations, is the first observation of a bulk induced odd-frequency superconducting state in a TI. Our results demonstrate that the experimental phenomenology of superconductivity at TI interfaces is richer than previously thought, and it highlights the potential of TI-SC heterostructures for realizing novel electronic states. Odd-frequency superconducting components may be of particular importance for the theoretical description of TI and semiconductor-based Majorana heterostructures which operate in a nonzero magnetic field.

The authors thank A. M. Black-Schaffer, R. M. Geilhufe, and J. Linder for helpful discussions. This work is based on experiments performed at the Swiss Muon Source $(\mathrm{S} \mu \mathrm{S})$, Paul Scherrer Institute, Villigen, Switzerland. J. A. K. and Z.S. are supported by the Swiss National Science Foundation (SNF-Grant No. 200021_165910). The work of A. V. B. and A. P. is supported by VILLUM FONDEN via the Centre of Excellence for Dirac Materials (Grant No. 11744), Knut and Alice Wallenberg Foundation (Grant No. KAW 2018.0104), and the European Research Council ERC-2018-SyG HERO. T. H. acknowledges the John Fell Oxford University Press (OUP) Research Fund, and thanks $\mathrm{RCaH}$ for their hospitality and Liam J. Collins-McIntyre and Liam B. Duffy for help with the MBE growth.

*Corresponding author. avb@nordita.org

${ }^{\dagger}$ Corresponding author.

zaher.salman@psi.ch

[1] M. Tinkham, Introduction to Superconductivity: Second Edition, Dover Books on Physics (Dover Publications, Mineola, NY, 2004).

[2] V. L. Berezinskii, Pis'ma Zh. Eksp. Teor. Fiz. 20, 628 (1974), http://www.jetpletters.ac.ru/ps/1792/article_27363 .shtml.

[3] T. R. Kirkpatrick and D. Belitz, Phys. Rev. Lett. 66, 1533 (1991).

[4] D. Belitz and T. R. Kirkpatrick, Phys. Rev. B 46, 8393 (1992).

[5] A. Balatsky and E. Abrahams, Phys. Rev. B 45, 13125 (1992).

[6] J. Linder and A. V. Balatsky, Rev. Mod. Phys. 91, 045005 (2019).
[7] Y. Tanaka, Y. Tanuma, and A. A. Golubov, Phys. Rev. B 76, 054522 (2007).

[8] Y. Tanaka, Y. Asano, A. A. Golubov, and S. Kashiwaya, Phys. Rev. B 72, 140503(R) (2005).

[9] Y. Asano, A. A. Golubov, Y. V. Fominov, and Y. Tanaka, Phys. Rev. Lett. 107, 087001 (2011).

[10] S. Higashitani, H. Takeuchi, S. Matsuo, Y. Nagato, and K. Nagai, Phys. Rev. Lett. 110, 175301 (2013).

[11] S. Mironov, A. Mel'nikov, and A. Buzdin, Phys. Rev. Lett. 109, 237002 (2012).

[12] Y. V. Fominov, Y. Tanaka, Y. Asano, and M. Eschrig, Phys. Rev. B 91, 144514 (2015).

[13] Y. Asano and A. Sasaki, Phys. Rev. B 92, 224508 (2015).

[14] A. Di Bernardo, Z. Salman, X. Wang, M. Amado, M. Egilmez, M. Flokstra, A. Suter, S. Lee, J. H. Zhao, T. Prokscha et al., Phys. Rev. X 5, 041021 (2015).

[15] L. Fu and C. L. Kane, Phys. Rev. Lett. 100, 096407 (2008).

[16] Y. Asano and Y. Tanaka, Phys. Rev. B 87, 104513 (2013).

[17] V. Stanev and V. Galitski, Phys. Rev. B 89, 174521 (2014).

[18] H. Ebisu, K. Yada, H. Kasai, and Y. Tanaka, Phys. Rev. B 91, 054518 (2015).

[19] M. Snelder, A. A. Golubov, Y. Asano, and A. Brinkman, J. Phys. Condens. Matter 27, 315701 (2015).

[20] T. J. Jackson, T. M. Riseman, E. M. Forgan, H. Glückler, T. Prokscha, E. Morenzoni, M. Pleines, C. Niedermayer, G. Schatz, H. Luetkens et al., Phys. Rev. Lett. 84, 4958 (2000).

[21] A. Suter, E. Morenzoni, N. Garifianov, R. Khasanov, E. Kirk, H. Luetkens, T. Prokscha, and M. Horisberger, Phys. Rev. B 72, 024506 (2005).

[22] P. Bakule and E. Morenzoni, Contemp. Phys. 45, 203 (2004).

[23] A. Yaouanc and P. de Réotier, Muon Spin Rotation, Relaxation, and Resonance: Applications to Condensed Matter, International Series of Monographs on Physics (OUP Oxford, Oxford, UK, 2011).

[24] T. Prokscha, E. Morenzoni, K. Deiters, F. Foroughi, D. George, R. Kobler, A. Suter, and V. Vrankovic, Nucl. Instrum. Methods Phys. Res., Sect. A 595, 317 (2008).

[25] E. Morenzoni, H. Glückler, T. Prokscha, R. Khasanov, H. Luetkens, M. Birke, E. M. Forgan, C. Niedermayer, and M. Pleines, Nucl. Instrum. Methods Phys. Res., Sect. B 192, 254 (2002).

[26] M. S. Pambianchi, J. Mao, and S. M. Anlage, Phys. Rev. B 50, 13659 (1994).

[27] M. G. Flokstra, R. Stewart, N. Satchell, G. Burnell, H. Luetkens, T. Prokscha, A. Suter, E. Morenzoni, S. Langridge, and S. L. Lee, Phys. Rev. Lett. 120, 247001 (2018).

[28] E. Morenzoni (private communication).

[29] See the Supplemental Material at http://link.aps.org/ supplemental/10.1103/PhysRevLett.125.026802 for additional sample characterization measurements, details of the theoretical modeling and further description of analysis and experimental methods. The Supplemental Material also includes the additional Refs. [30-39].

[30] L. J. Collins-McIntyre, M. D. Watson, A. A. Baker, S. L. Zhang, A. I. Coldea, S. E. Harrison, A. Pushp, A. J. Kellock, S. S. P. Parkin, G. van der Laan et al., AIP Adv. 4, 127136 (2014).

[31] H. Saadaoui, Z. Salman, T. Prokscha, A. Suter, B. M. Wojek, and E. Morenzoni, Phys. Procedia 30, 164 (2012). 
[32] A. Suter and B. M. Wojek, Phys. Procedia 30, 69 (2012).

[33] A. Suter, E. Morenzoni, N. Garifianov, R. Khasanov, E. Kirk, H. Luetkens, T. Prokscha, and M. Horisberger, Physica (Amsterdam) (374-375)B, 243 (2006).

[34] H. Wang, H. Liu, C.-Z. Chang, H. Zuo, Y. Zhao, Y. Sun, Z. Xia, K. He, X. Ma, X. C. Xie et al., Sci. Rep. 4, 5817 (2014).

[35] S. Wiedmann, A. Jost, B. Fauqué, J. van Dijk, M. J. Meijer, T. Khouri, S. Pezzini, S. Grauer, S. Schreyeck, C. Brüne et al., Phys. Rev. B 94, 081302 (2016).

[36] L. R. Doolittle, Nucl. Instrum. Methods Phys. Res., Sect. B 9, 344 (1985).

[37] M. Björck and G. Andersson, J. Appl. Crystallogr. 40, 1174 (2007).

[38] A. M. Black-Schaffer and A. V. Balatsky, Phys. Rev. B 87, 220506(R) (2013).

[39] A. Pertsova and A. Balatsky (to be published).

[40] E. Tsymbal, J. Magn. Magn. Mater. 130, L6 (1994).

[41] M. Lindstrom, B. Wetton, and R. Kiefl, J. Eng. Math. 85, 149 (2014).
[42] J. A. Krieger, Y. Ou, M. Caputo, A. Chikina, M. Döbeli, M.-A. Husanu, I. Keren, T. Prokscha, A. Suter, C.-Z. Chang et al., Phys. Rev. B 99, 064423 (2019).

[43] A. M. Black-Schaffer and A. V. Balatsky, Phys. Rev. B 86, 144506 (2012).

[44] T. Yokoyama, Phys. Rev. B 86, 075410 (2012).

[45] S.-Y. Xu, N. Alidoust, I. Belopolski, A. Richardella, C. Liu, M. Neupane, G. Bian, S.-H. Huang, R. Sankar, C. Fang et al., Nat. Phys. 10, 943 (2014).

[46] G. Rosenberg and M. Franz, Phys. Rev. B 85, 195119 (2012).

[47] C. Triola, J. Cayao, and A. M. Black-Schaffer, Ann. Phys. (Berlin) 532, 1900298 (2020).

[48] L. Fu and E. Berg, Phys. Rev. Lett. 105, 097001 (2010).

[49] T. Mizoguchi and M. Ogata, J. Phys. Soc. Jpn. 84, 084704 (2015).

[50] J. Schmidt, F. Parhizgar, and A. M. Black-Schaffer, Phys. Rev. B 101, 180512(R) (2020). 